\title{
Preface
}
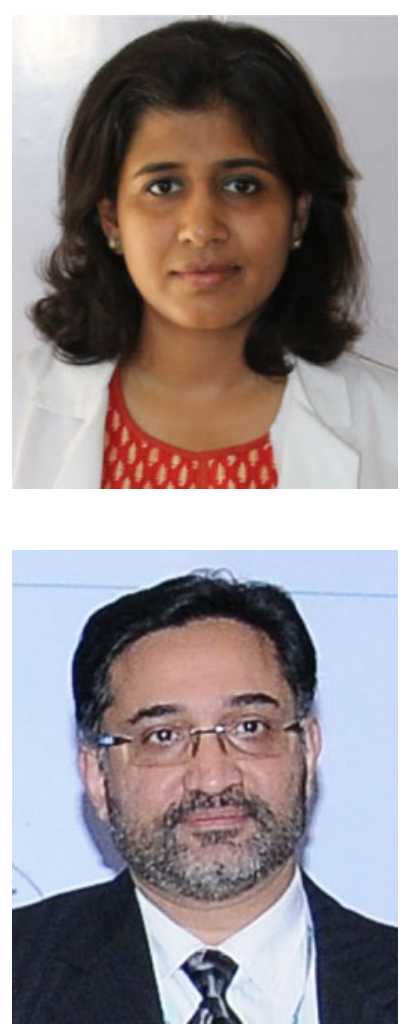

Peritoneal surface oncology is an emerging specialty in India. Cytoreductive surgery (CRS) and HIPEC have become popular worldwide for treating peritoneal metastases (PM) from various malignancies over the last couple of decades. This treatment can cure certain selected patients with PM and substantially prolong life and/or improve the quality of life in others. In India, most of the experience dates back to the last 5 years. The first workshop on peritoneal surface malignancies, in India, was held under the aegis of the Peritoneal Surface Oncology Group International (PSOGI) from the 16-18th of April,2015, at Fortis Hospital Bangalore, an initiative taken by Dr. Paul Sugarbaker. This 3 day program was attended by 70 surgical oncologists from all over the country in various stages of their careers. The program director Dr. Sugarbaker along with other experts in the field, Prof David Morris, Prof Olivier Glehen and Dr. Diane Goere delivered lectures on all aspects of CRS and HIPEC. The workshop also included a live surgery demonstration by Dr. Sugarbaker.
Most Indian surgeons have no formal training in CRS and HIPEC. It is not a part of the curriculum in surgical oncology training programs across the country. Treatment of PM is challenging and is associated with a prolonged learning curve. Along with the technical skills needed to perform the procedure, appropriate selection of patients is important for attaining optimal results. This workshop provided a comprehensive overview of the current practices for treatment of PM and was a starting point for those who wished to pursue this speciality further. The Indian surgeons had a unique opportunity to interact with the experts for 3 days and learn from their experience.

This workshop also had presentations by Indian surgeons practicing CRS and HIPEC about their experience. The PSOGI is a collaborative group of international experts in peritoneal surface oncology. The collaborative efforts of these experts over the years have been elemental in generating evidence and laying down guidelines for management of patients with PM from common and rare tumors both. There are such collaborative groups in France, Canada and the United states like the American Society of Peritoneal Surface Malignancies, the French surgical society AFC (AssociationFrançaise de Chirurgie), FROGHI (FRench Oncologic and Gynecologic HIPEC Group) and the Canadian HIPEC collaborative group.There are some national and international registries for peritoneal malignancies as well. The meeting was an opportunity for the Indian surgeons to interact with each other and collaborate and the first collaborative paper on the Indian experience with CRS and HIPEC is published in this issue.

The Indian Journal of Surgical Oncology (IJSO) is the official journal of the Indian Association of Surgical Oncology (IASO). During the workshop, Dr. Sugarbaker and Dr. KS Gopinath, the editor in chief of the IJSO conceived the idea of a special issue of the journal based on the presentations made during the meeting, which would be useful in educating a larger number of oncologists about the current standards of care and progress in the field of peritoneal surface oncology and commemorate this meeting. This special issue titled 'Proceedings of the meeting of the $1^{\text {st }}$ PSOGI workshop on Peritoneal Surface Malignancies (India)' is thus published here.

\section{Aditi Bhatt}

Sahbber Zaveri 